\title{
Evaluation of HuoXueHuaYu therapy for nonalcoholic fatty liver disease: a systematic review and meta-analysis of randomized controlled trial
}

Yunfei Cai ${ }^{1 \dagger}$, Qiuer Liang ${ }^{1 \dagger}$, Weihao Chen ${ }^{1+}$, Minghao Chen ${ }^{2,3}$, Ruixue Chen ${ }^{4}$, Yun Zhang ${ }^{1}$, Ya Xiao ${ }^{1 *}$ and Liguo Chen ${ }^{1 *}$

\begin{abstract}
Background: To evaluate the effectiveness and safety of HuoXueHuaYu (HXHY) therapy in treating nonalcoholic fatty liver disease (NAFLD) through a systematic review and meta-analysis.

Methods: We performed comprehensive searches on Embase, Pubmed, Cochrane Library, CNKI, VIP and Wanfang databases up to June 2017 for randomized controlled trials using HXHY in the treatment of NAFLD compared with conventional treatment.

Results: This meta-analysis included 13 studies involving 1429 patients which 775 patients belonged to HXHY group and 654 patients belonged to conventional treatment group. The results of meta-analysis showed that HXHY can significantly improve B ultrasonic level $(\mathrm{OR}=2.33 ; 95 \% \mathrm{Cl}: 1.60,3.40 ; P<0.00001)$ of NAFLD compared with conventional treatment. As to lipids, HXHY was tested to be better on reduction of total cholesterol (TC) (MD $=-0.38,95 \% \mathrm{Cl}:-0.48,-0.29 ; P<0.00001)$ and triglyceride $(\mathrm{TG})(\mathrm{MD}=-0.31 ; 95 \% \mathrm{Cl}:-0.37,-0.24 ; P<0.00001)$ than conventional treatment. $\mathrm{HXHY}$ also had a greater beneficial effect on liver function in reducing alanine transaminase (ALT) (MD $=-1.69 ; 95 \% \mathrm{Cl}:-2.24,-1.14 ; P<0.00001)$ and aspartate transaminase (AST) (MD $=-22.53 ; 95 \% \mathrm{Cl}:-33.16,-11.90 ; P<0.00001)$ compared with conventional treatment. $\mathrm{HXHY}$ can also significantly improve the effective rate $(\mathrm{OR}=3.55 ; 95 \% \mathrm{Cl}: 2.65,4.76 ; P<0.00001)$ compared with conventional treatment. No serious adverse reactions were reported.
\end{abstract}

Conclusions: HXHY seems to be an effective and safe therapy for NAFLD. It is suggested that further study of HXHY in the treatment of NAFLD requires trials with rigorous design, multicenter, large-scale and high-quality worldwide.

Keywords: Huoxuehuayu, NAFLD, Meta-analysis

\section{Background}

Nonalcoholic fatty liver disease (NAFLD) is a common chronic liver disease, with prevalence between 14 and $45 \%$ in the world [1,2]. One clinical study suggested that about $1 / 3$ of NAFLD patients could develop into nonalcoholic steatohepatitis (NASH), and once they developed into NASH, the risks of liver cirrhosis, liver cancer, and liver failure might increase significantly [3].

\footnotetext{
*Correspondence: xiaoya0527@126.com; tchenly@jnu.edu.cn

†Yunfei Cai, Qiuer Liang and Weihao Chen contributed equally to this work

'School of Traditional Chinese Medicine, Jinan University, Guangzhou, China Full list of author information is available at the end of the article
}

The main therapies of NAFLD in conventional treatment are lifestyle intervention and drug therapy. Lifestyle intervention is hard to be applied due to lack of compliance. Therefore, drug therapy plays an important role in treating patients with NAFLD. Vitamin E and pioglitazone showed positive effects on liver function and lipid deposition. However, in spite of some beneficial effects, vitamin $\mathrm{E}$ does not have therapeutic effect on liver fibrosis and pioglitazone causes weight gain [4]. Other drugs such as metformin, orlistat and statins were of limited benefit [5]. Therefore, development of an effective therapy is of significant importance for NAFLD. 
In Traditional Chinese medicine (TCM), chronic liver diseases are usually considered to be accompanied by blood stasis [6, 7]. Promoting blood circulation (Chinese name in pinyin "Huo Xue HuaYu" (HXHY)) is an important therapy in the treatment of NAFLD [8]. A previous study compared different TCM therapies for NAFLD and indicated that HXHY therapy was superior to other therapies in treating patients with NAFLD [9]. More and more traditional Chinese herbs with the function of activating blood circulation have been proved to be effective in treating NAFLD [10]. Though there are several clinical trials suggested that HXHY therapy has therapeutic potential in treating NAFLD, the effectiveness of HXHY has not been assessed in system. Therefore, the present meta-analysis aimed to evaluate the effectiveness and safety of HXHY in treating NAFLD by a systematic review and meta-analysis of randomized controlled trial (RCTs) to provide evidence for clinical practice.

\section{Methods}

\section{Search strategy}

The study was performed following the PRISMA guidelines [11]. The literature search was conducted using Cochrane Library (1993 to June 2017), the PubMed database (2000 to June 2017), the Embase database (1974 to June 2017), the China National Knowledge Infrastructure database (1979 to June 2017), the Wanfang database (1982 to June 2017), the VIP database (1989 to June 2017). Search terms were (NAFLD OR nonalcoholic fatty liver disease OR fatty liver disease) AND (HuoXueHuaYu OR activating blood circulation OR Chinese medicine OR herbs OR herbal medicine).

\section{Study selection}

Inclusion criteria were as following: (a) Patients were diagnosed with NAFLD; (b) The trial was claimed to be a RCT; (c) The formula used in the study included HXHY-class herbs. The herbs which have the function of activating blood circulation were defined as HXHY-class herbs. The most commonly used HXHY-class herbs in clinical practice are Salvia miltiorrhiza (Dan shen), Ligusticum wallichii (Chuan xiong), Hawthorn (Shan zha), Rhizoma curcumae longae (Jiang huang), Curcuma aromatic (Yu jin), Panax pseudo-ginseng (Tian qi), Peach kernel (Tao ren), Rhizoma sparganii (San leng), Curcuma zedoaria (E zhu), Carthami Flos (Hong hua), Eupatorium japonicum (Ze lan), Corydalis Rhizoma (Yan hu suo), Semen vaccariae (Wang bu. liu xing), etc.; (d) The study compared the efficacy of HXHY with conventional treatment.

Exclusion criteria were as following: (a) duplicated or redundant study; (b) nonhuman studies; (c) nonrandomized controlled trials.

\section{Outcome indicators}

The primary outcome was the level of type-B ultrasonic of liver, and the secondary outcomes were levels of total cholesterol (TC), triglyceride (TG), alanine transaminase (ALT) and aspartate transaminase (AST) and the effective rate.

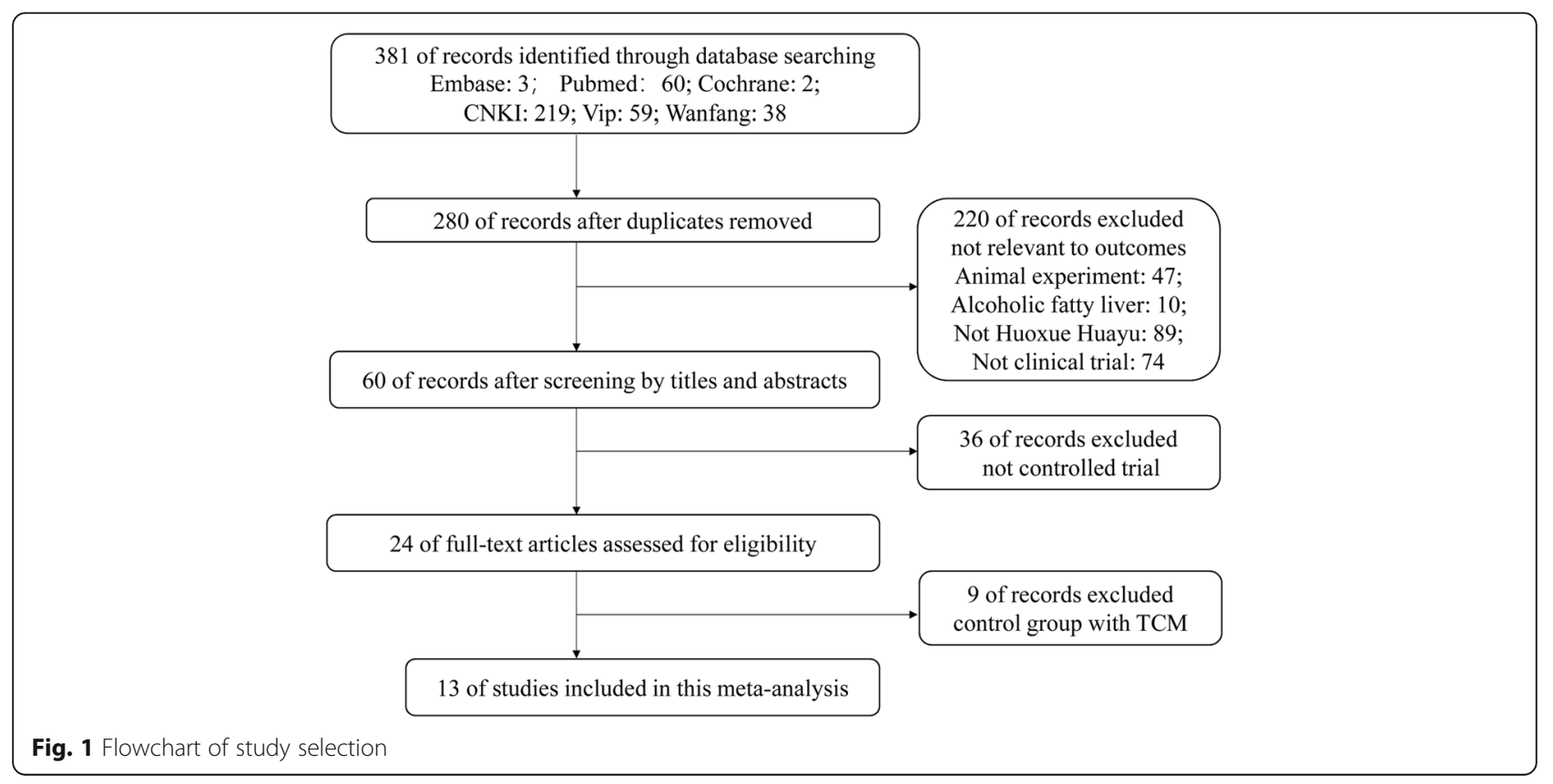




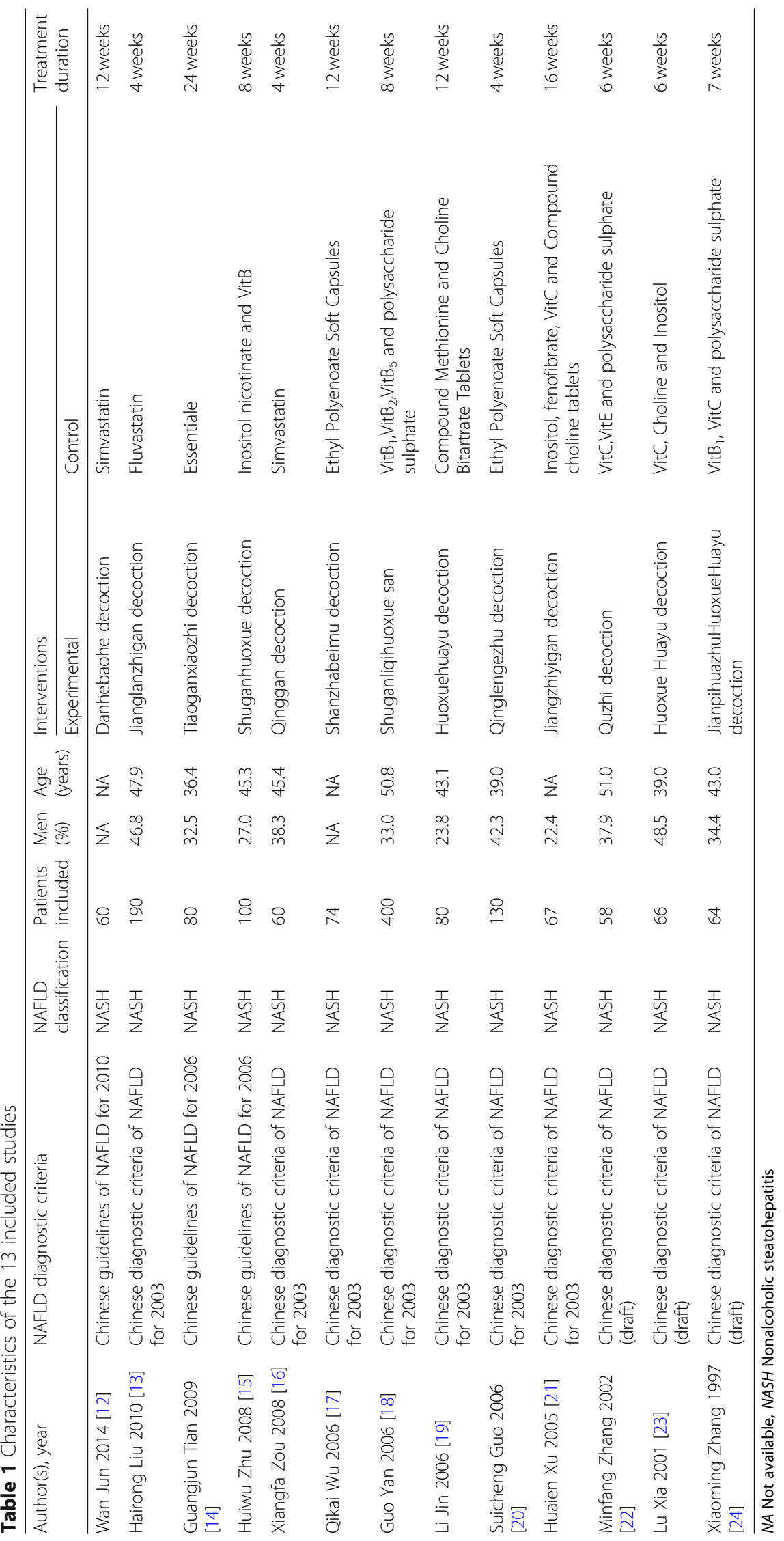




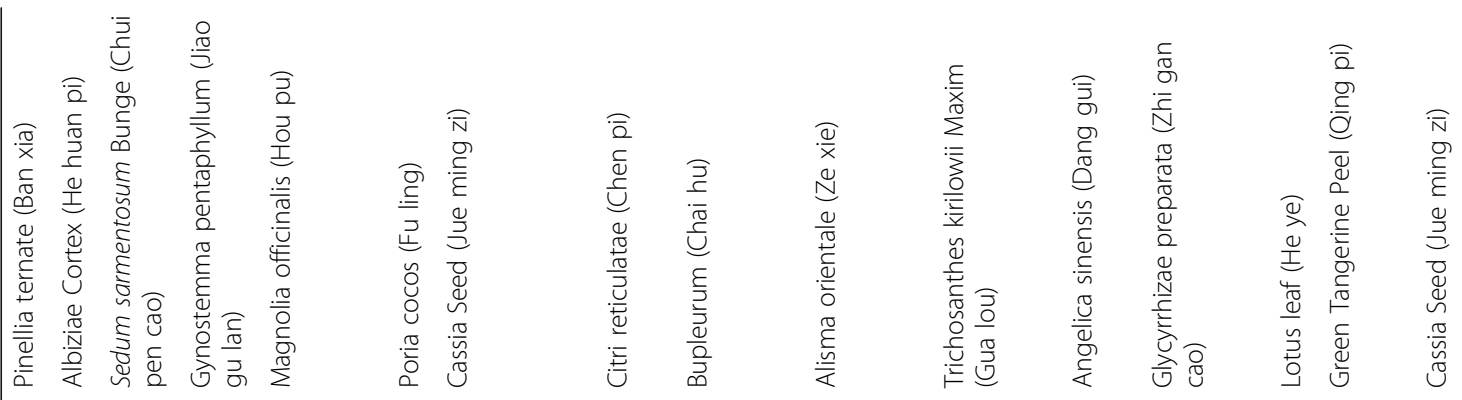

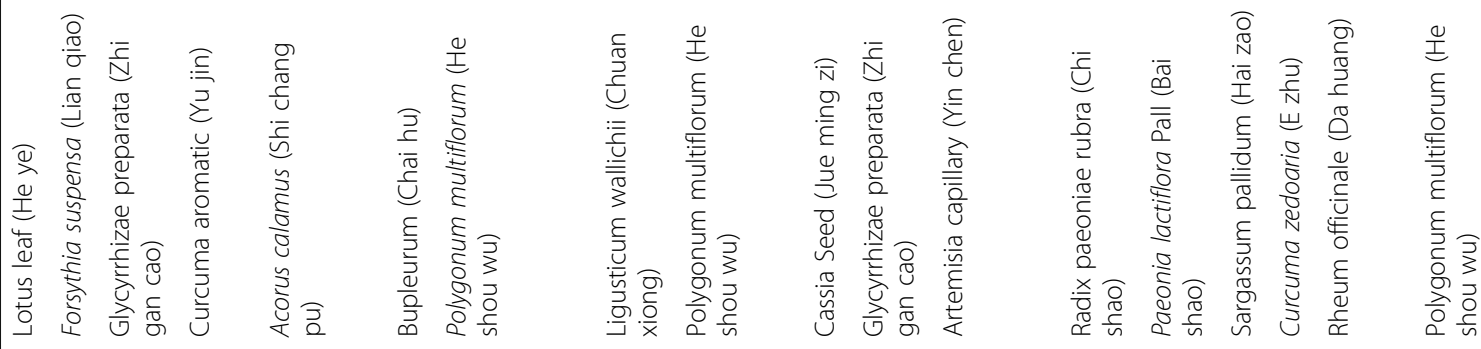

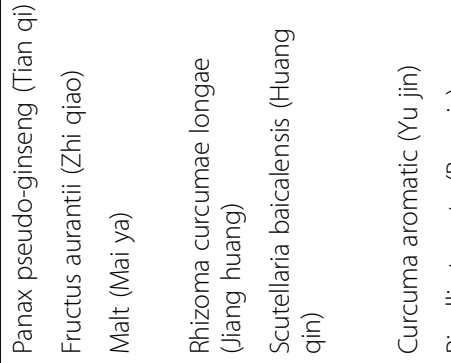

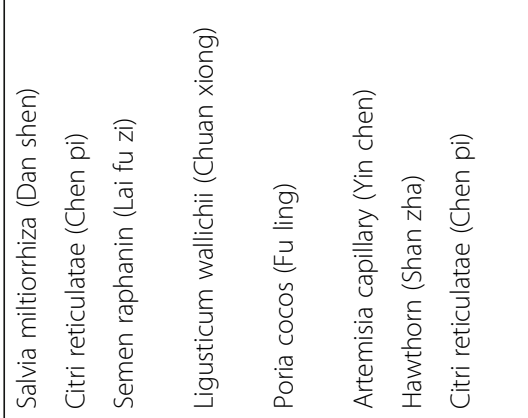

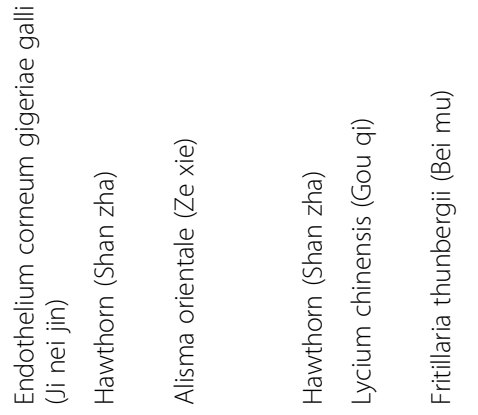

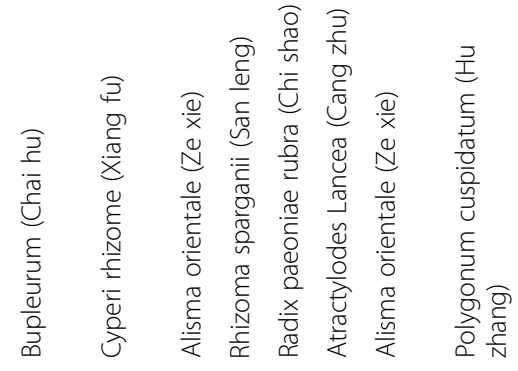




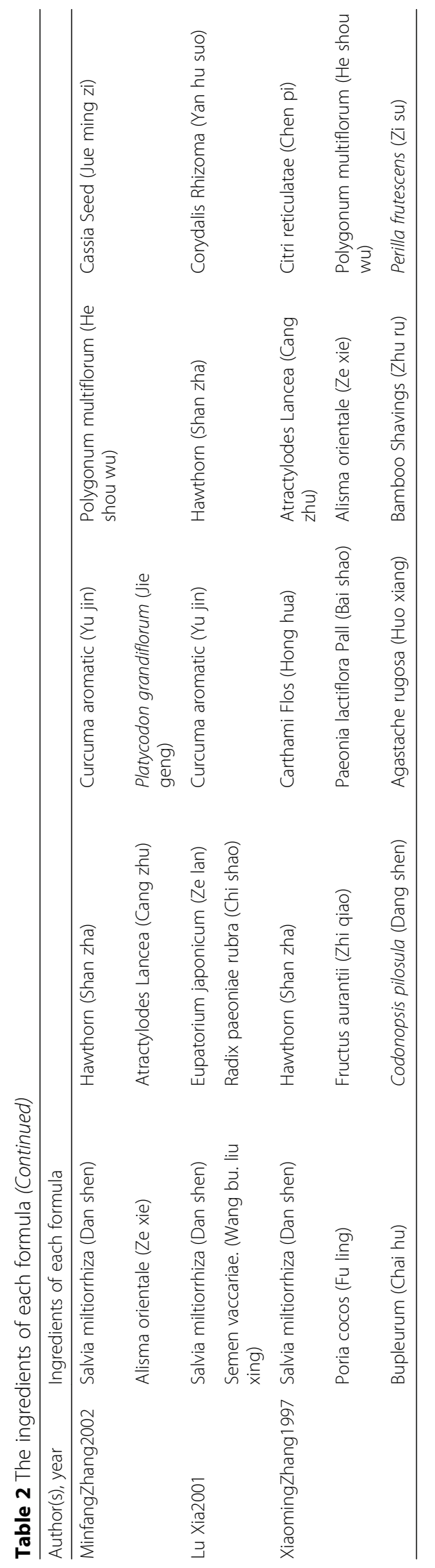




\section{Data extraction and analysis}

Data Extraction of the included studies were performed by two researchers independently. They discussed and recorded any disagreement. The third researcher resolved the disagreement that could not be resolved through discussion. Cochrane Risk of Bias Tool was used to evaluate the quality of RCTs.

Mean difference (MD) was reported for TC, TG, AST and ALT. Odds ratio (OR) was reported for B ultrasonic level and effective rate using Review Manager software (RevMan 5.3). 95\% confidence interval (CI) will be used as an effective size for the combined analysis. $I^{2}$ statistics is used to estimate heterogeneities. If there is no heterogeneity $\left(I^{2}<50 \%\right.$ and $\left.P>0.1\right)$, a fixed-effect model is used to synthesize the data; Otherwise, if there is heterogeneity $\left(50 \%<I^{2}<75 \%\right)$, a random-effect model was applied. When $I^{2}>75 \%$, subgroup analysis or sensitivity analysis was performed to identify the causes of the heterogeneity. A funnel plot was selected to assess the publication bias.

\section{Results}

\section{Study selection and quality evaluation}

Firstly, we searched out 381 studies completely and then keep 280 studies after deleting repeated records. Eliminating case reports, reviews and animal researches, we achieved 13 studies [12-24] (Fig. 1). The 13 studies included 1429 cases in total. Of which, 775 belonged to the HXHY therapy group and 654 belonged to the conventional treatment group. The patients included in each study were all classified as NASH. Table 1 listed the characteristics of the studies. Table 2 listed the compositions of the herbal formulae.

Figure 2 showed the quality evaluation of the included studies. In terms of random sequence generation, only 1 study used a table of random number [12], while 12 studies mentioned "random", but there was no detail of the randomization method. In the aspect of blinding, no studies mentioned blinding of the patients and personnel. In addition, no study reported allocation concealment. Selective reporting, incomplete outcome data and blinding of outcome assessment were evaluated as low risk of bias.

\section{The effect of HuoXueHuaYu therapy on B ultrasonic level in patients with NAFLD}

Seven studies reported type-B ultrasonic. These studies involved 590 patients including 327 patients in HXHY therapy group and 263 patients in the control group. We found no significant heterogeneity in these studies ( $\left.\mathrm{I}^{2}=0 \%, \quad \mathrm{P}=0.85\right)$. A fixed effects model analysis showed that HXHY was more beneficial to change type$B$ ultrasonic level in NAFLD Patients when compared to

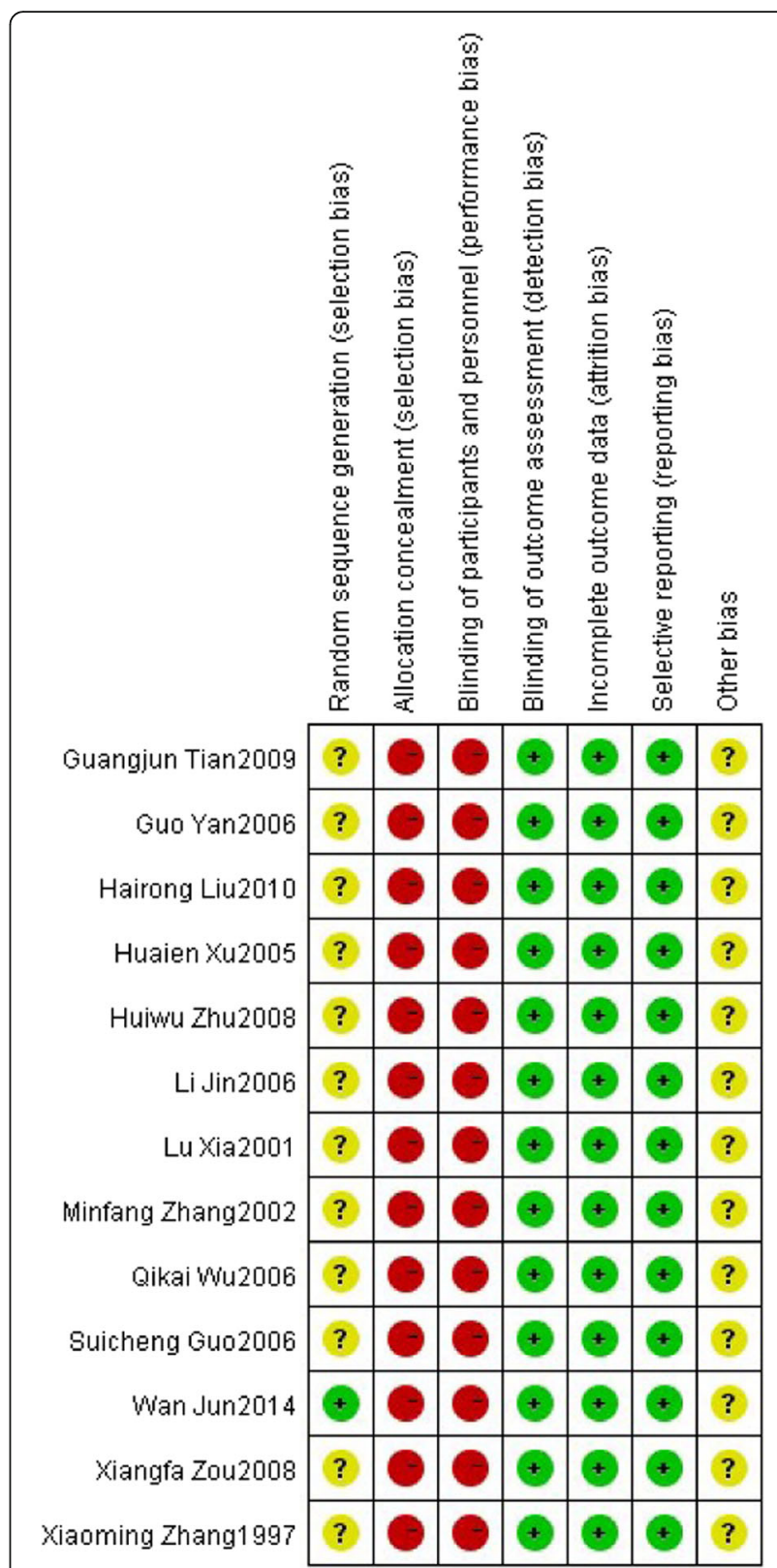

Fig. 2 Potential risk of bias of each included study

the conventional treatment group $(\mathrm{OR}=2.33 ; 95 \% \mathrm{CI}$ : 1.60, 3.40; $P<0.0001$ ) (Fig. 3).

The effect of HuoXueHuaYu therapy on blood lipids in patients with NAFLD

Five studies reported total cholesterol (TC). These studies including 358 patients which 202 patients belonged to the HXHY therapy group and 156 patients belonged to the conventional treatment group. As the results showed, the heterogeneity of TC was high $\left(I^{2}=94 \%, P<0.00001\right)$ among trials when comparing HXHY therapy with conventional treatment group. The random-effect model analysis 


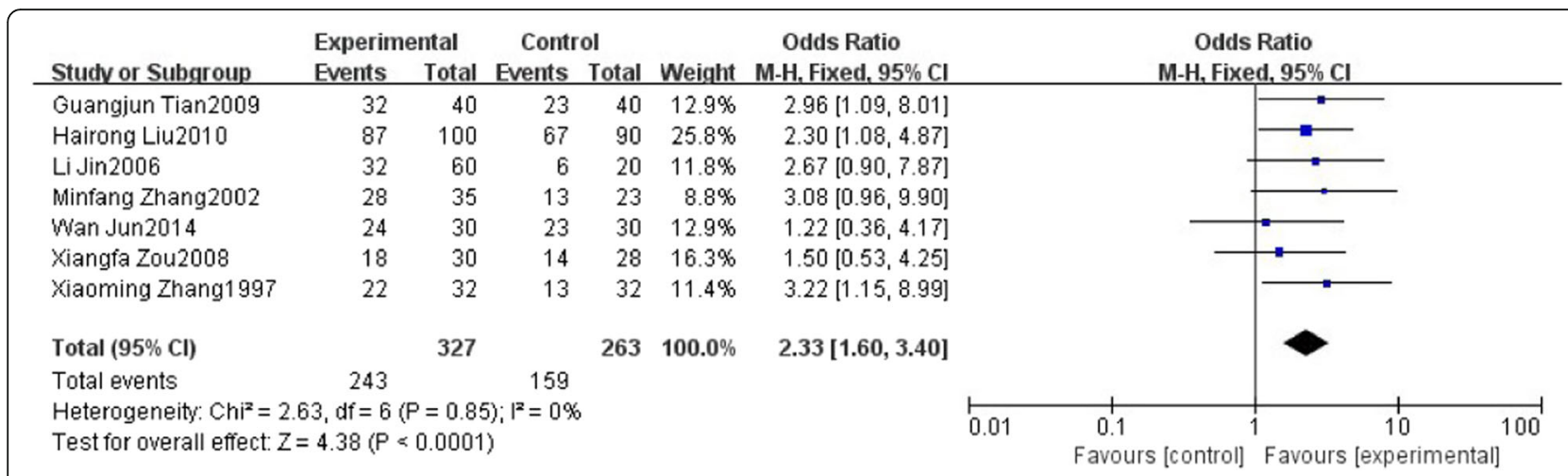

Fig. 3 The effect of HXHY therapy on B ultrasonic level in NAFLD patients

showed that patients with NAFLD who received HXHY had significantly lower TC levels than those who received conventional therapy. ( $\mathrm{MD}=-0.38$; 95\% CI: $-0.48,-0.29$; $P<0.00001$ ) (Fig. 4a). Sensitivity analysis results suggested that the study carried out by Guangjun Tian 2009 made a great contribution to the high heterogeneity. There was no heterogeneity existed when the study was removed $\left(I^{2}=0 \%\right.$, $P=0.73$ ) (Fig. 4b). Meanwhile, we found that the duration of the study was 24 weeks which was obviously longer than other studies, indicating that the duration maybe a source of heterogeneity.

Six studies reported triglyceride (TG). These studies including 418 patients which 232 patients belong to the HXHY therapy group and 186 patients belong to the conventional treatment group. We found high significant heterogeneity in TG $\left(I^{2}=95 \%, P<0.00001\right)$ among trials when comparing HXHY therapy with conventional treatment group. A random-effect model analysis showed that HXHY therapy significantly decrease the level of TG than conventional treatment $(\mathrm{MD}=-0.31 ; 95 \% \mathrm{CI}$ $-0.37,-0.24 ; P<0.00001$ ) (Fig. 5a). Sensitivity analysis results suggested that the study carried out by Guangjun Tian. 2009 made a great contribution to the high heterogeneity. The heterogeneity was much smaller when this study was removed. $\left(I^{2}=49 \%, P=0.10\right)$ (Fig. $\left.5 \mathrm{~b}\right)$.

\section{The effect of HuoxueHuayu therapy on liver function in patients with NAFLD}

Six studies reported alanine transaminase (ALT). These studies including 418 patients which 232 patients belong

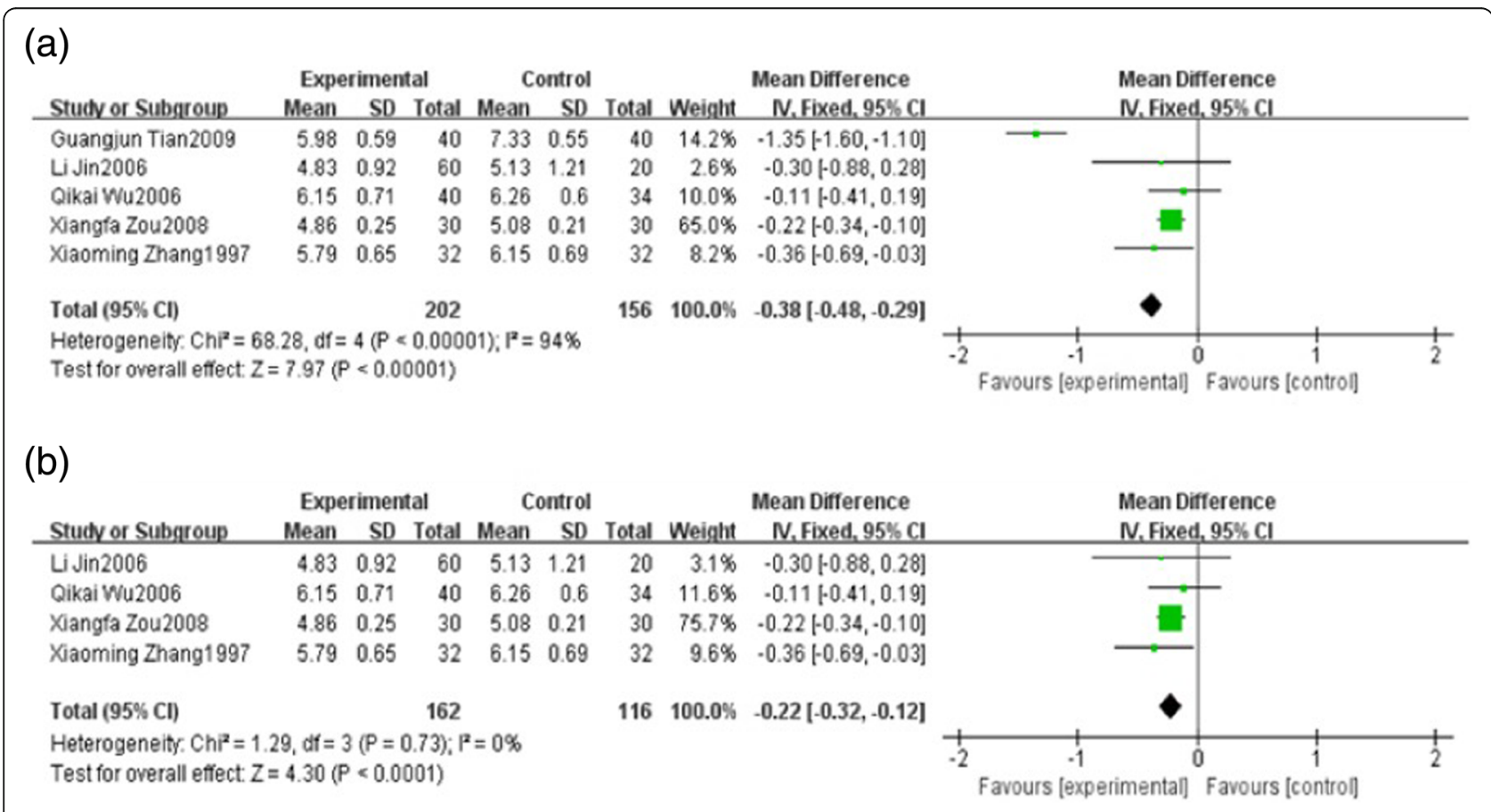

Fig. 4 a The effect of HXHY on blood lipid total cholesterol in NAFLD patients. b Sensitivity analysis was performed by omitting one study 


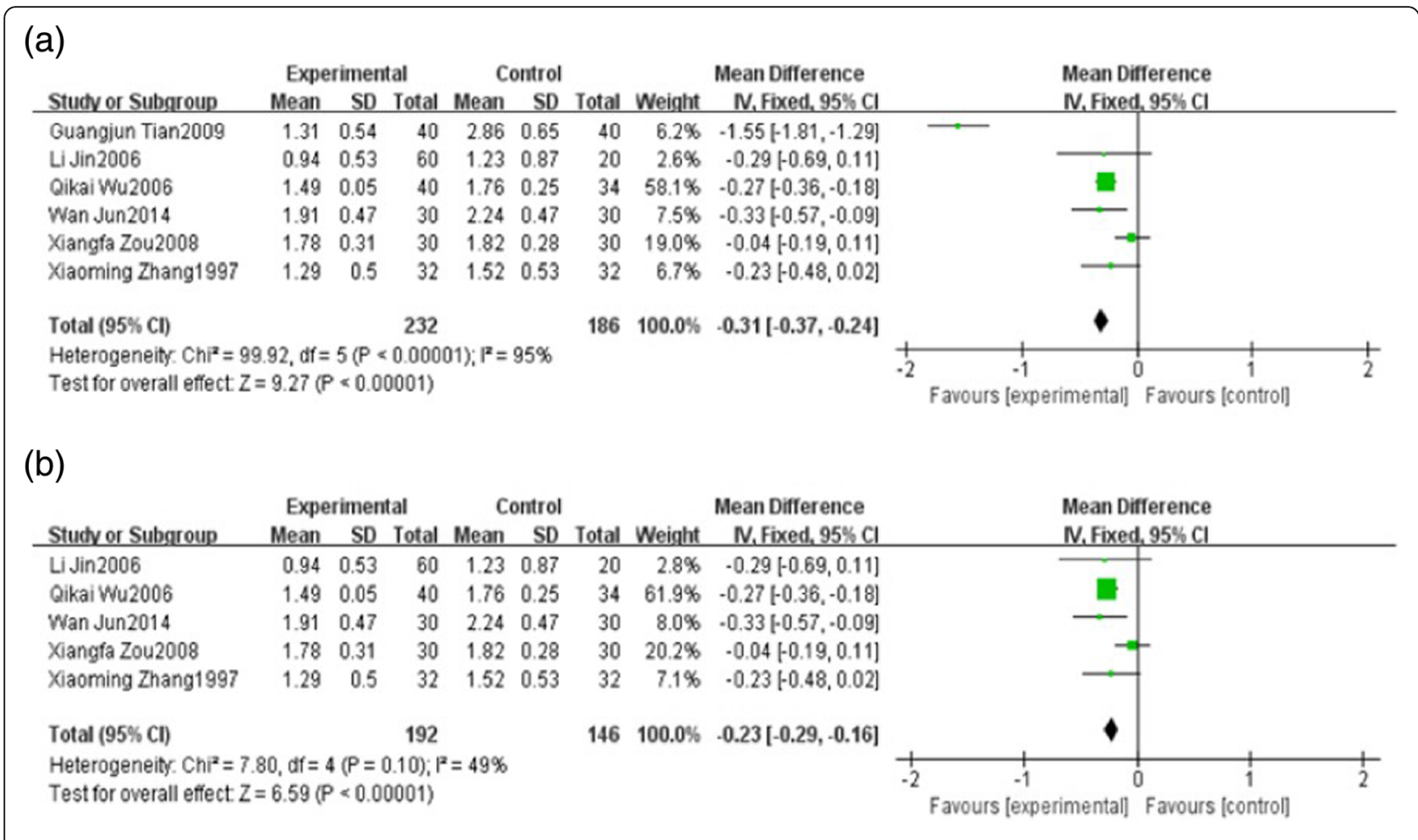

Fig. 5 a The effect of HXHY on blood lipid triglyceride in NAFLD patients. b Sensitivity analysis was performed by omitting one study

to the HXHY therapy group and 186 patients belong to the conventional treatment group. As the results showed, we found high significant heterogeneity in ALT $\left(I^{2}=82 \%, P<0.0001\right)$ among trials when comparing HXHY therapy with conventional treatment group. A random-effect model analysis showed that HXHY therapy significantly reduce the level of ALT than conventional treatment in the NAFLD patients $(\mathrm{MD}=-1.69$; 95\% CI: $-2.24,-1.14 ; P<0.00001$ ) (Fig. 6). Although we conducted sensitivity analysis and subgroup analysis, there was still a high heterogeneity.

Five studies reported aspartate transaminase (AST). We found high significant heterogeneity in AST $\left(I^{2}=97 \%, P<\right.$ 0.00001) among trials when comparing HXHY with conventional treatment group. A random-effect model analysis showed that HXHY significantly reduce the level of
AST than conventional treatment in the NAFLD patients $(\mathrm{MD}=-22.53$; 95\% CI:- 33.16, $-11.90 ; P<0.0001)$ (Fig. 7). Although we conducted sensitivity analysis and subgroup analysis, there was still a high heterogeneity.

\section{The effect of HuoXueHuaYu therapy on the effective rate} in patients with NAFLD

Twelve studies reported effective rate of HuoxueHuayu therapy in patients with NAFLD. These studies including 1369 patients which 745 patients belong to the therapy group and 624 patients belong to the conventional treatment group. The effective rate in seven studies [13, 18, 20-23] refers to the proportion of participants with improvement of clinical symptoms and level of type-B ultrasonic of liver. The effective rate in the other five studies [12, 14, 17, 19, 24]

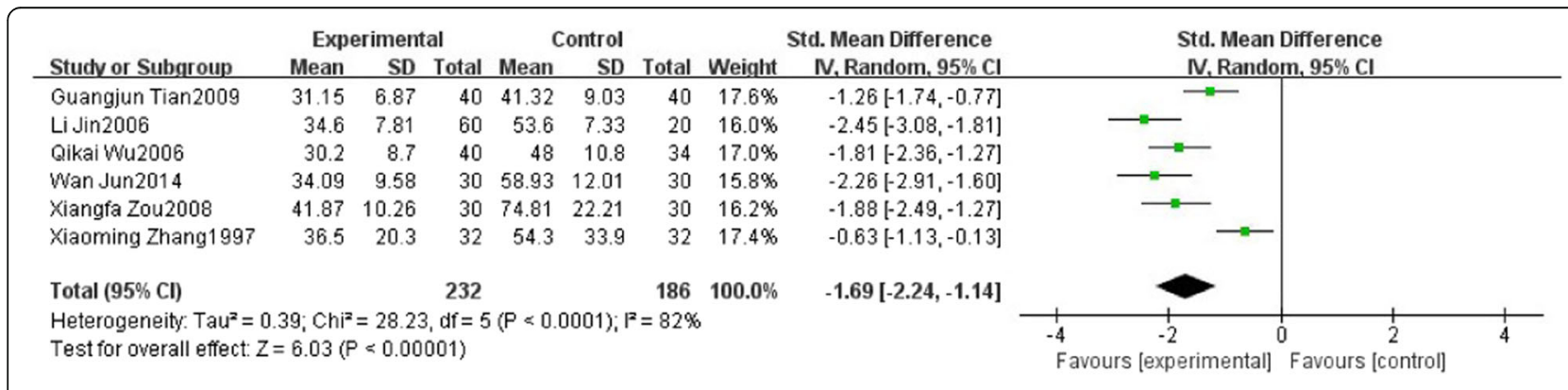

Fig. 6 The effect of HXHY on alanine transaminase (ALT) in NAFLD patients 


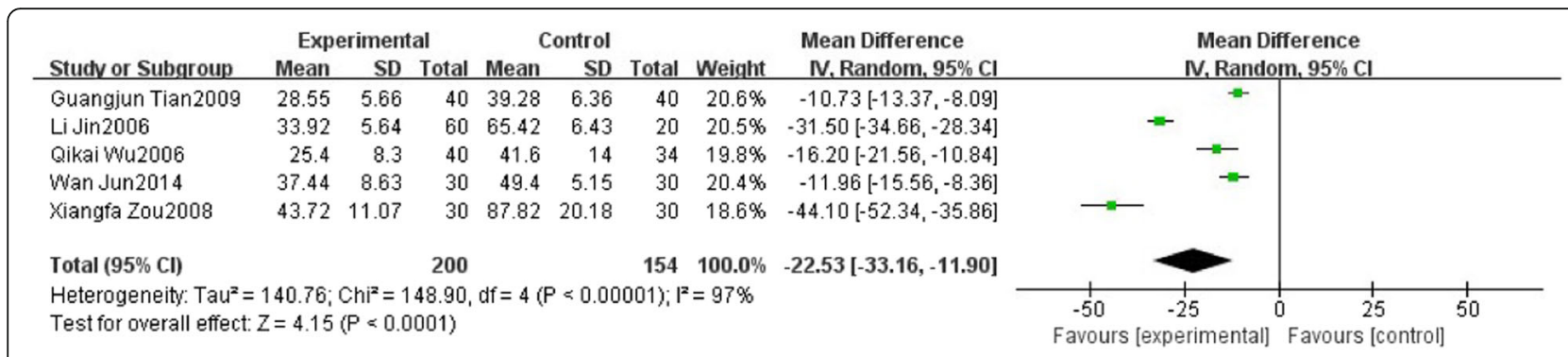

Fig. 7 The effect of HXHY on aspartate transaminase (AST) in NAFLD patients

refers to the proportion of participants with improvement of clinical symptoms and level of type-B ultrasonic of liver as well as $\geq 30 \%$ reduction in level of liver function and blood lipids. There was no significant heterogeneity with $\mathrm{I}^{2}=0 \%, \quad P=0.99$. A fixed effects model analysis showed that HXHY was more beneficial for the effective rate in NAFLD Patients when compared with the conventional treatment group $(\mathrm{OR}=3.55$; 95\% $\mathrm{CI}: 2.65$, 4.76; $P<$ 0.00001) (Fig. 8). Funnel plot was selected to assess the publication bias for the effective rate, which showed that the distribution is generally almost symmetrical (Fig. 9).

\section{The safety evaluation of HuoxueHuayu therapy on NAFLD patients}

There were no adverse reactions reported in all the 13 articles. Therefore, we may need to assess the safety of HXHY therapy on NAFLD in facilitate further researches.

\section{Discussion}

Based on the meta-analysis of 13 RCTs, it can be documented that HXHY can significantly improve B ultrasound in NAFLD patients when compared with the conventional treatment group. Meanwhile, HXHY therapy also can improve the blood lipid, liver function and the effective rate. Furthermore, there was no obvious adverse reaction reported in treating NAFLD. Our results suggested that HXHY is effective and safe in treating NAFLD.

The mechanism of NAFLD is not fully understood. Recently, researches show that insulin resistance, free radicals and oxidative stress, endoplasmic reticulum stress, and inflammation may involve in the mechanism of NAFLD [25-28]. The general philosophical underlining of Chinese medicine is holistic medicine $[29,30]$. TCM has anti-inflammatory effect and high safety in treating chronic liver diseases [31, 32]. Previous studies suggested that HXHY therapy can protect hepatic cells, improve liver function and control the development of hepatic fibrosis. For example, salvianolic acid B extracted from Radix Salvia miltiorrhiza were demonstrated to attenuate liver damage, hepatic steatosis, and reduce the levels of pro-inflammatory cytokines [33]. Hawthorn leaf flavonoids significantly lowered liver/body weight ratio, improved serum parameters and liver dysfunction and alleviated hepatic lipid accumulation [34].

\begin{tabular}{|c|c|c|c|c|c|c|c|c|c|}
\hline \multirow{2}{*}{$\begin{array}{l}\text { Studv or Subgroup } \\
\text { Guangjun Tian2009 }\end{array}$} & \multicolumn{2}{|c|}{ Experimental } & \multicolumn{2}{|c|}{ Control } & Weight & $\begin{array}{c}\text { Odds Ratio } \\
\text { M-H, Fixed, } 95 \% \mathrm{Cl}\end{array}$ & \multicolumn{3}{|c|}{$\begin{array}{c}\text { Odds Ratio } \\
\text { M-H, Fixed, } 95 \% \mathrm{Cl}\end{array}$} \\
\hline & 37 & 40 & 28 & 40 & $4.2 \%$ & $5.29[1.36,20.53]$ & & & \\
\hline Guo Yan2006 & 176 & 200 & 138 & 200 & $33.2 \%$ & $3.29[1.96,5.55]$ & & & 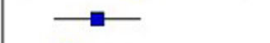 \\
\hline Hairong Liu2010 & 87 & 100 & 67 & 90 & $18.4 \%$ & $2.30[1.08,4.87]$ & & & \\
\hline Huaien Xu2005 & 36 & 38 & 23 & 29 & $2.7 \%$ & $4.70[0.87,25.29]$ & & & \\
\hline Huiwu Zhu2008 & 45 & 50 & 34 & 50 & $6.8 \%$ & $4.24[1.41,12.70]$ & & & \\
\hline Li Jin2006 & 54 & 60 & 13 & 20 & $3.9 \%$ & $4.85[1.39,16.87]$ & & & \\
\hline LuXia2001 & 38 & 40 & 20 & 26 & $2.4 \%$ & $5.70[1.05,30.87]$ & & & \\
\hline Minfang Zhang 2002 & 33 & 35 & 17 & 23 & $2.3 \%$ & $5.82[1.06,32.00]$ & & & \\
\hline Qikai Wu2006 & 34 & 40 & 20 & 34 & $6.5 \%$ & $3.97[1.31,11.97]$ & & & \\
\hline Suicheng Guo2006 & 74 & 80 & 38 & 50 & $7.0 \%$ & $3.89[1.36,11.19]$ & & & \\
\hline Wan Jun2014 & 27 & 30 & 22 & 30 & $4.4 \%$ & $3.27[0.77,13.83]$ & & & \\
\hline Xiaoming Zhang199? & 22 & 32 & 13 & 32 & $8.1 \%$ & $3.22[1.15,8.99]$ & & & \\
\hline Total $(95 \% \mathrm{Cl})$ & & 745 & & 624 & $100.0 \%$ & $3.55[2.65,4.76]$ & & & \\
\hline Total events & 663 & & 433 & & & & & & \\
\hline $\begin{array}{l}\text { Heterogeneity: } \mathrm{Chi}^{2}=2 \\
\text { Test for overall effect: } Z\end{array}$ & $\begin{array}{l}39, \mathrm{df}=11 \\
=8.51(\mathrm{P}=\end{array}$ & $\begin{array}{l}(P=0.9 \\
0.0000\end{array}$ & 19); $\left.\right|^{2}=0$ & & & & 0.05 & $\begin{array}{cc}1 & 1 \\
\text { Favours [control] } & 1\end{array}$ & $\begin{array}{lcc}1 & 5 & 1 \\
1 & 20 \\
\text { Favours [experimental] }\end{array}$ \\
\hline
\end{tabular}




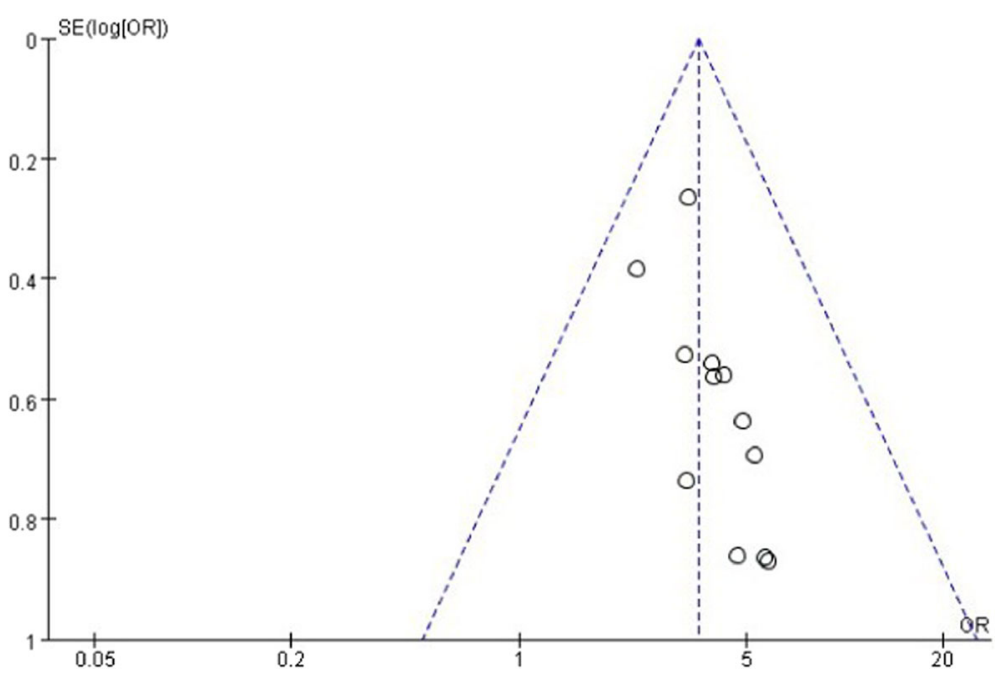

Fig. 9 The funnel plot of HXHY therapy on the effective rate in NAFLD patients

Our study had some limitations. First, the quality of the included trials was generally not high. None of the studies provided the methods of blinding and allocation concealment [35]. Clinical trials should be reported in accordance with the Consolidated Standards of Reporting Trials (CONSORT) standards [36, 37]. Second, the treatment duration of most studies was short. Because NAFLD is chronic disease, longer duration should be taken to assess the safety and effectiveness of HXHY in the treatment of NAFLD. The sensitivity analysis suggested that treatment duration may be the main source of heterogeneity. Third, the sample size of some of the included studies is small. It is necessary to demonstrate whether the effects of HXHY will not be changed in future large-scale trials. Fourth, a wide variety of drugs were used in the control group across the studies, which may be another important source of the heterogeneity. Despite the limitations of this study, to the best of our knowledge, this is the first study to evaluate HXHY therapy for NAFLD.

\section{Conclusions}

In conclusion, this study indicates that HXHY therapy is more effective compared with conventional treatment for patients with NAFLD, suggesting that HXHY may be a new option for treating NAFLD. Due to the pool quality of the included studies, it is necessary to validate the conclusions by more rigorously designed, multi-centered RCTs with high quality.

\section{Abbreviations}

ALT: Alanine transaminase; AST: Aspartate transaminase; Cl: Confidence interval; CNKI: Chinese journal full-text database; HXHY: HuoXueHuaYu; MD: Mean difference; NAFLD: Nonalcoholic fatty liver disease;

NASH: Nonalcoholic steatohepatitis; OR: Odds ratio; RCTs: Randomized controlled trials; TC: Total cholesterol; TCM: Traditional Chinese medicine; TG: Triglyceride
Acknowledgements

Not applicable.

Authors' contributions

LC and YX contributed in study design. YC and QL contributed in study search and selection. MC, RC and $Y Z$ contributed in data extraction and analysis. YC and $Y X$ contributed in drafting the paper. QL, YX and WC contributed in revising the paper. All authors approved the final version to be published.

\section{Funding}

This work was supported by the National Natural Sciences Foundation of China (No. 81673848 and 81603520), the Natural Sciences Foundation of Guangdong province (No. 2016A030310084 and 2017A030313658), the Administration of Traditional Medicine of Guangdong province (No. 20161063 and No. 20181068), the Fundamental Research Funds for the Central Universities (No. 21616315), the Medical Scientific Research Foundation of Guangdong Province (No. 2016111221315850) and the Science \& Technical Plan of Guangzhou, Guangdong, China (No. 201707010100 and NO.201804010213). The funding bodies play no role in the design of the study and collection, analysis, and interpretation of data and in writing the manuscript.

Availability of data and materials

All data generated or analysed during this study are included in this published article.

Ethics approval and consent to participate

Not applicable.

\section{Consent for publication}

Not applicable.

\section{Competing interests}

The authors declare that they have no competing interests.

\section{Author details}

${ }^{1}$ School of Traditional Chinese Medicine, Jinan University, Guangzhou, China. ${ }^{2}$ Division of Histology and Embryology, Key Laboratory for Regenerative Medicine of the Ministry of Education, Medical College, Jinan University, Guangzhou, China. ${ }^{3}$ International Joint Laboratory for Embryonic Development and Prenatal Medicine, Medical College, Jinan University, Guangzhou, China. ${ }^{4}$ Second Affiliated Hospital, Guangzhou Medical University, Guangzhou, China. 
Received: 17 January 2018 Accepted: 11 July 2019

Published online: 19 July 2019

\section{References}

1. Li Y, Wang J, Tang Y, Han X, Liu B, Hu H, et al. Bidirectional association between nonalcoholic fatty liver disease and type 2 diabetes in Chinese population: evidence from the Dongfeng-Tongji cohort study. PLoS One. 2017;12(3):e174291.

2. Bellentani S, Marino M. Epidemiology and natural history of non-alcoholic fatty liver disease (NAFLD). Ann Hepatol. 2009;8(Suppl 1):S4-8.

3. Tziomalos K, Athyros VG, Paschos P, Karagiannis A. Nonalcoholic fatty live disease and statins. Metabolism. 2015;64(10):1215-23.

4. EASL-EASD-EASO. Clinical practice guidelines for the management of nonalcoholic fatty liver disease. J Hepatol. 2016;64(6):1388-402.

5. Gitto S, Vitale G, Villa E, Andreone P. Treatment of nonalcoholic steatohepatitis in adults: present and future. Gastroenterol Res Pract. 2015;2015:732870

6. Zhang Z, Li Q, Li J. Consensus on traditional Chinese medicine treatment of nonalcoholic fatty liver disease. Beijing J Tradit Chin Med. 2011;30(2):83-6.

7. Qiu R. The application of activating blood circulation to dissipate blood stasis in chronic disease. Traditional Chin Med Res. 2003;16(5):44-5.

8. Sun L, Zhao AJ, Wei YC, Li ZF, Wang LP, Liu TT. Research Progress of Huo Xue Hua Yu therapy in the treatment of nonalcoholic fatty liver disease. Mod J Integ Trad Chin West Med. 2017;26(3):339-42.

9. He JS, Gao H, Shao L, Tong GD, Chen L, Zhang L, et al. The clinical study of different therapies of TCM for nonalcoholic fatty liver disease. Clin J Trad Chin Med. 2010;22(6):499-501.

10. Peng $H$, He Y, Zheng G, Zhang W, Yao Z, Xie W. Meta-analysis of traditional herbal medicine in the treatment of nonalcoholic fatty liver disease. Cell Mol Biol (Noisy-le-Grand). 2016;62(4):88-95.

11. Liberati A, Altman DG, Tetzlaff J, Mulrow C, Gotzsche PC, loannidis JP, et al. The PRISMA statement for reporting systematic reviews and meta-analyses of studies that evaluate health care interventions: explanation and elaboration. J Clin Epidemiol. 2009;62(10):e1-e34.

12. Wan J, Zhang X. Clinical observation on XiaojiHuatan and HuoxueHuayu therapy treating 30 patients with nonalcoholic fatty liver disease. Med Inf. 2014;27(4):111-2.

13. Liu H. Clinical observation on Jianglan Zhigan decoction in the treatment of 100 cases with nonalcoholic fatty liver disease. Jiangsu J Tradit Chin Med. 2010;42(2):32-3.

14. Tian G, Chi X, Chen Q, Rui W, Xiao H. Clinical observation on Tiaogan Xiao zhi decoction in the treatment of 40 cases with nonalcoholic fatty liver disease. Henan Tradit Chin Med. 2009:29(4):374-5.

15. Zhu H. Effect of Shugan Huayu Qutan therapy in the treatment of 50 cases with nonalcoholic fatty liver disease. Zhejiang J Integ Trad West Med. 2008;18(7):426-7.

16. Zou X, Chen F, Wang S. Effect of Qinggan decoction in the treatment of 30 cases with nonalcoholic fatty liver disease. Shanxi Tradit Chin Med. 2008;29(1):20-1

17. Wu Q, Fan Q, Cheng J. Effect of Shanzha Beimu decoction in the treatment of 40 cases with nonalcoholic fatty liver disease. Chin J Integ Tradit West MedDiges. 2006;14(1):54-5.

18. Guo Y, Zhu S, Bao Q, Li N, Fang Q. Effect of treatment with Quzhi Rougan san in patients with nonalcoholic fatty liver disease. Mod J Integ Trad Chin West Med. 2006;15(10):1300-1.

19. Li J. Clinical observation on Huoxuehuayu and Huatanlishi therapy in the treatment of 60 cases with nonalcoholic fatty liver disease. Zhejiang Clin Med. 2006:8(4):375.

20. Guo S. Clinical observation on Huoxuehuayu therapy in the treatment of 80 cases with nonalcoholic fatty liver disease. Shanxi Traditional Chin Med. 2006:27(11):1355-6.

21. $\mathrm{Xu} \mathrm{H}$. Clinical observation on Jiangzhi Yigan decoction in the treatment of 38 cases with nonalcoholic fatty liver disease. Henan Tradit Chin Med. 2005;25(10):44-5.

22. Zhang M, Li H, Wang S. Clinical observation on Quzhi decoction in the treatment of 35 cases with nonalcoholic fatty liver disease. Shaanxi Trad Chin Med. 2002;23(10):903-4.

23. Lu X, Yi C. Clinical observation on Huoxue Huayu therapy in the treatmen of 40 cases with nonalcoholic fatty liver disease. Fujian J Trad Chin Med. 2001;32(6):32.
24. Zhang X. Clinical observation on Jianpihuazhuo and Huoxuehuayu therapy in the treatment of 32 cases with nonalcoholic steatohepatitis. Chin J Integ Tradit West Med Liver Dis. 1997;7(2):119-20.

25. Wei $X$, Wang $C$, Hao S, Song H, Yang L. The therapeutic effect of Berberine in the treatment of nonalcoholic fatty liver disease: a meta-analysis. Evid Based Complement Alternat Med. 2016;2016:3593951.

26. Abenavoli L, Greco M, Milic N, Accattato F, Foti D, Gulletta E, et al. Effect of Mediterranean diet and antioxidant formulation in non-alcoholic fatty liver disease: a randomized study. Nutrients. 2017;9(8). https://doi.org/10.3390/ nu9080870

27. Fisher FM, Kim M, Doridot L, Cunniff JC, Parker TS, Levine DM, et al. A critical role for ChREBP-mediated FGF21 secretion in hepatic fructose metabolism. Mol Metab. 2017:6(1):14-21.

28. Privitera G, Spadaro L, Alagona C, Calanna S, Piro S, Rabuazzo AM, et al. Hepatic insulin resistance in NAFLD: relationship with markers of atherosclerosis and metabolic syndrome components. Acta Diabetol. 2016:53(3):449-59.

29. Hsiao $\mathrm{WL}$, Liu L. The role of traditional Chinese herbal medicines in cancer therapy--from TCM theory to mechanistic insights. Planta Med. 2010:76(11):1118-31.

30. Porter D, Cochrane S, Zhu X. Current usage of traditional Chinese medicine for breast Cancer-a narrative approach to the experiences of women with breast Cancer in Australia-a pilot study. Medicines (Basel). 2017;4(2). https:// doi.org/10.3390/medicines4020020.

31. Chien CF, Wu YT, Tsai TH. Biological analysis of herbal medicines used for the treatment of liver diseases. Biomed Chromatogr. 2011;25(1-2):21-38.

32. Liu ZL, Xie LZ, Zhu J, Li GQ, Grant SJ, Liu JP. Herbal medicines for fatty liver diseases. Cochrane Database Syst Rev. 2013;8:D9059.

33. Zeng W, Shan W, Gao L, Gao D, Hu Y, Wang G, et al. Inhibition of HMGB1 release via salvianolic acid B-mediated SIRT1 up-regulation protects rats against non-alcoholic fatty liver disease. Sci Rep. 2015;5:16013.

34. Li Z, Xu J, Zheng P, Xing L, Shen H, Yang L, et al. Hawthorn leaf flavonoids alleviate nonalcoholic fatty liver disease by enhancing the adiponectin/ AMPK pathway. Int J Clin Exp Med. 2015;8(10):17295-307.

35. Berger W. Randomization, permuted blocks, masking, allocation concealment, and selection bias in the tobacco quit line study. Contemp Clin Trials. 2010;31(3):201

36. Campbell MK, Piaggio G, Elbourne DR, Altman DG. Consort 2010 statement: extension to cluster randomised trials. BMJ. 2012;345:e5661.

37. Xiao L, Hu J, Zhang L, Shang HC. Endorsement of CONSORT by Chinese medical journals: a survey of "instruction to authors". Chin J Integr Med. 2014:20(7):510-5.

\section{Publisher's Note}

Springer Nature remains neutral with regard to jurisdictional claims in published maps and institutional affiliations.
Ready to submit your research? Choose BMC and benefit from:

- fast, convenient online submission

- thorough peer review by experienced researchers in your field

- rapid publication on acceptance

- support for research data, including large and complex data types

- gold Open Access which fosters wider collaboration and increased citations

- maximum visibility for your research: over $100 \mathrm{M}$ website views per year

At BMC, research is always in progress.

Learn more biomedcentral.com/submissions 